\title{
Investigation of Elastic Properties of Aorta in Patients with Coronary Slow Flow
}

\author{
Macit Kalçık ${ }^{1}$, Mahmut Yesin ${ }^{2}$, Ahmet Güner ${ }^{3}$,Emrah Bayam ${ }^{4}$, Tolga Doğan ${ }^{1}$, \\ Mucahit Yetim ${ }^{1}$, Lütfü Bekar ${ }^{1}$, Oğuzhan Çelik ${ }^{5}$
}

${ }^{1}$ Department of Cardiology, Hitit University Faculty of Medicine, Çorum, Turkey

${ }^{2}$ Department of Cardiology, Kars Harakani State Hospital, Kars, Turkey

${ }^{3}$ Department of Cardiology, Kartal Kosuyolu Heart Training and Research Hospital, Istanbul, Turkey

${ }^{4}$ Department of Cardiology, Umraniye Training and Research Hospital, Istanbul, Turkey

${ }^{5}$ Department of Cardiology, Muğla Sıtkı Koçman University Training and Research Hospital, Muğla, Turkey

Correspondence:

Macit Kalçık

Address: Department of Cardiology, Hitit University Faculty of Medicine, Çorum,Turkey

Email: macitkalcik@yahoo.com

Received: 19.03.2019,

Accepted: 30.05 .2018

https://doi.org/10.5799/jcei/5834

\section{ABSTRACT}

Objective: Coronary slow flow (CSF) phenomenon is characterized by delayed opacification of coronary vessels in a normal coronary angiogram. Although clinical and pathological features have been previously described, the underlying pathophysiology has not been fully understood yet. Aortic elastic properties have a predictive value in detecting early stages of atherosclerosis. In this study, we aimed to the evaluate the elastic properties of ascending aorta in patients with CSF.

Materials and Methods: This single-center study enrolled 50 angiographically identified patients with CSF (23 female, mean age: $51.6 \pm 9.7$ years), along with 50 controls (19 female, mean age: $53.5 \pm 8.7$ years) with normal coronary flow. The quantitative measurement of coronary blood flow was performed using the thrombolysis in myocardial infarction frame count method. Aortic elastic parameters including aortic strain, aortic distensibility, aortic stiffness index and aortic compliance were calculated using M-mode echocardiography derived aortic diameters using accepted formulae.

Results: There was no significant difference in terms of clinical, demographic, echocardiographic and laboratory parameters between the patients with CSF and the controls. The hemodynamic parameters including aortic pulse pressure, aortic mean pressure, aortic fractional pulse pressure and aortic pulsatility index were similar between patients with CSF and controls. Systolic and diastolic aortic diameters and pulsatile aortic diameter change were similar between the groups. There was also no significant difference in terms of aortic strain, aortic distensibility, aortic stiffness index and aortic compliance between the groups.

Conclusion: The results demonstrated that there was no significant difference in terms of hemodynamic parameters and elastic properties of ascending aorta between CSF patients and control group with a similar risk profile and demographic characteristics. Further large scale studies are required to determine the relationship between CSF and elastic properties of aorta.

Keywords: aortic stiffness index, coronary angiography, coronary slow flow, echocardiography

\section{INTRODUCTION}

Coronary slow flow (CSF) phenomenon is described as slow antegrade progression of contrast material to the distal branch of a coronary arteries in the absence of obstructive coronary artery disease [1]. The prevalence of CSF varies from 1 to $7 \%$ among patients undergoing coronary angiogram for stable angina pectoris [2]. CSF has been reported to be related with various clinical events, such as acute myocardial infarction, angina pectoris, lifethreatening arrhythmias, and sudden death
[3]. The exact mechanism associated with this interesting phenomenon remains largely unclear, but several mechanisms have been proposed including enhanced oxidative stress and inflammation, microvascular dysfunction, impaired endothelial functions, platelet function disorder and diffuse atherosclerosis [4-8].

Aortic stiffness is defined as the arterial rigidity caused by the loss of elastic tissue in the arterial wall that decreases the widening capacity of the artery. Damage to the arterial wall due to inflammation and 
atherosclerosis causes a decrease in arterial distensibility, compliance and elasticity [9]. Arterial stiffness is a marker of increased cardiovascular risk and a predictor for cardiovascular morbidity and mortality in various populations [10]. Furthermore cardiovascular risk factors have been shown to be positively associated with arterial stiffness and patients with angiographically documented coronary artery disease have been shown to have stiffer arteries than control subjects [11,12]. Non-invasive assessment of aortic elastic properties may provide a method for early detection of atherosclerotic changes. Many different indices have been developed for proper estimation of arterial stiffness such as aortic strain, distensibility, compliance, and stiffness index [13-15].

We hypothesized that the mechanisms in the pathogenesis of CSF may also play role in the development of aortic atherosclerosis and increased aortic stiffness. In this study, we aimed to the evaluate the elastic properties of ascending aorta in patients with CSF.

\section{MATERIALS AND METHODS}

\section{Study Population}

This single-center study enrolled 50 angiographically identified patients with normal coronary arteries and CSF (23 female, mean age: $51.6 \pm 9.7$ years), along with 50 angiographically normal coronary flow patients with a similar risk profile and demographic characteristics (19 female, mean age: $53.5 \pm 8.7$ years). Patients with diagnosed with coronary artery disease, history of myocardial infarction, left ventricular dysfunction (left ventricular ejection fraction $<50 \%$ ), severe heart valve disease, cardiomyopathy, arrhythmia, active infection, connective tissue disease and liver, kidney or thyroid dysfunction were excluded from the study. All patients underwent transthoracic echocardiography (TTE). Complete blood count and blood chemistry panel were carried out in all patients at the time of admission. All demographic, laboratory and echocardiographic parameters were recorded into a dataset and compared between CSF patients and controls. All patients provided a written informed consent and the study protocol was approved by the local ethics committee of the hospital in accordance with the Declaration of Helsinki and Good Clinical Practice guidelines.

\section{Echocardiography}

All patients were evaluated by TTE which was performed by using a $2.5-\mathrm{MHz}$ probe on a Vivid 7 Doppler echocardiographic unite (GE Vingmed Ultrasound, Horten, Norway) in the left lateral decubitus position. Parasternal long axis and short axis views and apical two, four and five chamber views were used during TTE evaluation. In all patients, left atrial diameter (LAD), left ventricular enddiastolic diameter (LVEDD), left ventricular end-systolic diameter (LVESD), interventricular septal thickness (IVST) and posterior wall thickness (PWT) were measured on the parasternal long-axis view. Left ventricular ejection fraction (LVEF) was calculated by using biplane Simpson's method.

\section{Laboratory Analysis}

In order to perform complete blood count and blood chemistry panel, venous blood samples were collected after 12-hours of fasting by a clean puncture of an antecubital vein from all patients. Complete blood countings were measured on Sysmex XT2000i analyser (Sysmex corporation, Kobe, Japan). Fasting blood glucose, urea, creatinine, aspartate transaminase (AST), alanine transaminase (ALT), sodium, potassium, uric acid, total cholesterol (TC), high-density lipoprotein (HDL), and triglyceride (TG) levels were also measured on an autoanalyzer (Siemens Advia 2400 Chemistry System, Siemens Diagnostic, Tarrytown, USA). Low-density lipoprotein (LDL), was calculated using the Friedewald formula [LDL $(\mathrm{mg} / \mathrm{dL})=\mathrm{TC}-(\mathrm{HDL}+\mathrm{TG} / 5)]$ [16].

\section{Coronary Angiography and the diagnosis of CSF}

Coronary angiography was done by clinical indications such as abnormal stress test results, positive treadmill test, dobutamine stress echo, typical chest pain, or signs of ischemia during myocardial perfusion scintigraphy. All study population underwent selective coronary artery angiography after appropriate patient preparation. Femoral artery and sometimes radial or brachial artery cannulation was used for the arterial access site and a Judkins system was applied for cannulation of the left and right coronary arteries. Coronary angiographies were evaluated by at least two independent interventional cardiologists. CSF was investigated by using the thrombolysis in myocardial infarction (TIMI) frame count (TFC) method described by Gibson et al [17].

The quantitative measurement of the coronary blood flow was performed by two cardiologists with no prior knowledge regarding the patients' diagnosis and condition by using the TFC method ${ }^{13}$. The starting point was considered as the moment the contrast material contacted both sides of the coronary artery and began to advance. The end point was considered as the moment when the contrast material reached the distal branching point, known as the moustache in the left anterior descending (LAD) coronary artery, appeared on the first side branch of the posterolateral artery in the right coronary artery (RCA), and could be imaged in the distal bifurcation of the longest branch of the circumflex $(\mathrm{Cx})$ coronary artery. As the LAD is notably longer than the other arteries, its measured TFC was divided by 1.7 (corrected TFC). By taking the exclusion criteria into account, patients with at least one coronary artery with a frame count above 36.2 for the LAD, 22.2 for the Cx and 20.4 for the RCA were determined as having CSF [18].

\section{Measurement of Aortic Pressures}

All patients had blood pressure measured prior to echocardiographic assessment. Aortic pressure measurements were performed while the patients were 
sitting comfortably on a chair with their feet stepping on the floor using a sphygmomanometer with an appropriately sized cuff (wrapping at least $80 \%$ of the forearm). Blood pressure measurements were done 12 hours after the last administration of a vasoactive drug or alcohol consumption and after 3 hours of abstinence of caffeine and tobacco. Patients rested 10 minutes in a supine position in a quiet room at a temperature of $20-22{ }^{\circ} \mathrm{C}$ before measurements were done. During measurements, the average of at least three measurements were taken into consideration for analysis of systolic (SBP) and diastolic blood pressures (DBP).

Calculation of hemodynamic parameters were performed using the formulae below:

- Aortic Pulse Pressure = SBP - DBP

- Aortic Mean Pressure $=[\mathrm{SBP}+(\mathrm{DBP} \times 2)] / 3$

- Aortic Fractional Pulse Pressure = Aortic Pulse Pressure / Aortic Mean Pressure.

- Aortic Pulsatility Index = Aortic Pulse Pressure / DBP.

\section{Measurement of Aortic Elastic Properties}

Aortic systolic (ASD) and diastolic diameters (ADD) were assessed on the basis of a 2D guided M-mode recording of the proximal ascending aorta, defined as $3 \mathrm{~cm}$ above the aortic valve in the parasternal long axis view with TTE. ASD was measured at the time of full opening of the aortic valve, and ADD was measured at the peak of the R wave of the simultaneously recorded electrocardiogram; five measurements were averaged for each diameter.

Aortic elastic parameters including aortic strain, aortic distensibility, aortic stiffness index and aortic compliance were calculated using the formulae below:

- Aortic Strain (\%) = 100 x [(ASD - ADD) / ADD]

- Aortic Distensibility $\left(10^{-6} \mathrm{~cm}^{2} \mathrm{dyn}^{-1}\right)=(2 \mathrm{x}$ Aortic Strain) / [100 x (SBP - DBP)]

- Aortic Stiffness Index = Logarithm $[100 \mathrm{x}(\mathrm{SBP} / \mathrm{DBP})$ / Aortic Strain]

- Aortic Compliance $(\mathrm{cm} / \mathrm{mmHg})=(\mathrm{ASD}-\mathrm{ADD}) /$ (SBP - DBP)

\section{Statistical Analysis}

Statistical analyses were performed using IBM SPSS Statistics for Windows, Version 19.0. (IBM Corp. Armonk, NY). The variables were investigated using analytical methods (Kolmogorov-Smirnov/Shapiro-Wilk test) to determine whether or not they were approximately normally distributed. Descriptive statistics were reported as mean with standard deviation for continuous variables with normal distribution, median and $25^{\text {th }}-75^{\text {th }}$ percentile values for continuous variables without normal distribution, and frequencies with percentages for the categorical variables. Group comparisons for continuous variables were tested using Student $\mathrm{t}$ test when data distribution was normal and using Mann-Whitney $U$ test when data distributions were
Table 1. Comparison of demographic and echocardiographic parameters between patient groups with and without coronary slow flow phenomenon

\begin{tabular}{lccc}
\hline Demographic Parameters & $\begin{array}{c}\text { CSF } \\
(\mathbf{n}=50)\end{array}$ & $\begin{array}{c}\text { Controls } \\
(\mathbf{n}=50)\end{array}$ & p value \\
\hline Age (years) & $51.6 \pm 9.7$ & $53.5 \pm 8.7$ & 0.309 \\
\hline Gender, (female), $\mathrm{n}(\%)$ & $23(46)$ & $19(38)$ & 0.418 \\
\hline Body Mass Index (kg/m $\left.{ }^{2}\right)$ & $28.3 \pm 5.1$ & $27.7 \pm 4.6$ & 0.740 \\
\hline Hypertension, $\mathrm{n}(\%)$ & $18(36)$ & $13(26)$ & 0.280 \\
\hline Diabetes Mellitus, $\mathrm{n}(\%)$ & $9(18)$ & $7(14)$ & 0.585 \\
\hline Dyslipidemia, $\mathrm{n}(\%)$ & $11(22)$ & $9(18)$ & 0.617 \\
\hline Smoking, $\mathrm{n}(\%)$ & $16(32)$ & $15(30)$ & 0.829 \\
\hline Family history of CAD, $\mathrm{n}(\%)$ & $8(16)$ & $10(20)$ & 0.603 \\
\hline Systolic BP (mmHg) & $118.8 \pm 12.6$ & $116.3 \pm 8.1$ & 0.236 \\
\hline Diastolic BP (mmHg) & $76.7 \pm 10.4$ & $75.2 \pm 7.5$ & 0.432 \\
\hline Heart Rate (bpm) & $78.7 \pm 13.5$ & $77.1 \pm 11.8$ & 0.269 \\
\hline Echocardiographic Parameters & & & \\
\hline Left atrial diameter (mm) & $32.2 \pm 4.3$ & $31.6 \pm 3.5$ & 0.252 \\
\hline LV ejection fraction (\%) & $63.3 \pm 3.6$ & $62.9 \pm 4.1$ & 0.584 \\
\hline LV end-diastolic diameter (mm) & $43.4 \pm 3.2$ & $42.3 \pm 4.7$ & 0.236 \\
\hline LV end-systolic diameter (mm) & $28.1 \pm 3.4$ & $27.4 \pm 4.7$ & 0.427 \\
\hline $\begin{array}{l}\text { Interventricular septal } \\
\text { thickness(mm) }\end{array}$ & $10.2 \pm 1.3$ & $9.6 \pm 1.2$ & 0.128 \\
\hline Posterior wall thickness (mm) & $9.9 \pm 1.2$ & $9.4 \pm 1.1$ & 0.133 \\
\hline $\begin{array}{l}\text { BP: Blood pressure, CAD: Coronary artery disease, CSF: Coronary slow flow, LV: Left } \\
\text { ventricle }\end{array}$ & & &
\end{tabular}

not normal. Comparisons for categorical variables were evaluated by Chi-square test. Significance level was accepted as $\mathrm{p}<0.05$ in all statistical analyses.

\section{RESULTS}

The clinical and demographical characteristics of patients with and without CSF were presented in Table 1. There was no significant difference between the groups in terms of age, gender, body mass index, frequency of diabetes mellitus, hypertension, dyslipidemia, smoking status and family history of coronary artery disease. Heart rate, SBP, and DBP were also similar between the groups. Comparison of echocardiographic parameters yielded that there was no significant difference in terms of LAD, LVEDD, LVESD, IVST, PWT and LVEF between CSF patients and controls.

Upon comparison of laboratory parameters between CSF group and controls, there was no significant difference in terms of white blood cells, platelets, hemoglobin, fasting blood glucose, urea, creatinine, AST, ALT, sodium, potassium, uric acid, TC, HDL, LDL, and TG levels between the groups (Table 2 ).

The TFC values were calculated separately for each coronary artery in two groups. The TFCs in LAD (45.4 \pm 2.9 vs. $20.3 \pm 1.8$ frames $), \mathrm{Cx}(39.2 \pm 4.3$ vs. $18.8 \pm 1.3$ frames $)$ and RCA ( $41.1 \pm 3.7$ vs. $19.7 \pm 1.6$ frames $)$ were significantly lower in CSF patients than the controls $(\mathrm{p}<0.001$ for all) (Table 3). 


\section{Aortic Elasticity in Coronary Slow Flow}

Table 2. Comparison of laboratory parameters between patient groups with and without coronary slow flow (CSF) phenomenon

\begin{tabular}{lccc}
\hline & $\begin{array}{c}\text { CSF } \\
(\mathbf{n}=50)\end{array}$ & $\begin{array}{c}\text { Controls } \\
(\mathbf{n}=50)\end{array}$ & $\begin{array}{c}\mathbf{P} \\
\text { value }\end{array}$ \\
\hline White blood cell $\left(\times 10^{3} / \mathrm{mL}\right)$ & $7.35 \pm 1.68$ & $7.46 \pm 2.41$ & 0.721 \\
\hline Neutrophils $\left(\times 10^{3} / \mathrm{mL}\right)$ & $4.89 \pm 1.68$ & $4.84 \pm 2.11$ & 0.826 \\
\hline Lymphocytes $\left(\times 10^{3} / \mathrm{mL}\right)$ & $1.63 \pm 0.69$ & $1.59 \pm 0.81$ & 0.841 \\
\hline Platelets $\left(\times 10^{3} / \mathrm{mL}\right)$ & $234.6 \pm 56.2$ & $229.4 \pm 52.7$ & 0.761 \\
\hline Hemoglobin $(\mathrm{mg} / \mathrm{dL})$ & $11.3 \pm 1.8$ & $11.8 \pm 1.6$ & 0.394 \\
\hline Glucose $(\mathrm{mg} / \mathrm{dL})$ & $103.5 \pm 24.3$ & $98.7 \pm 32.7$ & 0.542 \\
\hline Urea $(\mathrm{mg} / \mathrm{dL})$ & $15.7 \pm 5.1$ & $14.6 \pm 4.6$ & 0.246 \\
\hline Creatinine $(\mathrm{mg} / \mathrm{dL})$ & $1.0 \pm 0.1$ & $0.9 \pm 0.2$ & 0.127 \\
\hline $\begin{array}{l}\text { Aspartate transaminase } \\
\text { (u/L) }\end{array}$ & $28(21-43)$ & $30(19-46)$ & 0.493 \\
\hline $\begin{array}{l}\text { Alanine transaminase } \\
\text { (u/L) }\end{array}$ & $18(14-31)$ & $17(12-38)$ & 0.618 \\
\hline Sodium (mEq/L) & $136(135-139)$ & $137(134-140)$ & 0.756 \\
\hline Potassium (mEq/L) & $4.41 \pm 0.53$ & $4.27 \pm 0.49$ & 0.342 \\
\hline Uric acid (mg/dL) & $4.4 \pm 0.7$ & $4.6 \pm 0.9$ & 0.129 \\
\hline Total cholesterol (mg/dL) & $191(138-221)$ & $196(146-214)$ & 0.215 \\
\hline $\begin{array}{l}\text { High density lipoprotein } \\
\text { (mg/dL) }\end{array}$ & $43.7 \pm 9.1$ & $45.3 \pm 10.1$ & 0.491 \\
\hline $\begin{array}{l}\text { Low density lipoprotein } \\
\text { (mg/dL) }\end{array}$ & $117.3 \pm 19.2$ & $121.4 \pm 21.7$ & 0.198 \\
\hline Triglyceride (mg/dL) & $135.8 \pm 48.7$ & $157.6 \pm 56.6$ & 0.431 \\
\hline & & & \\
\hline
\end{tabular}

Table 3. Comparison of TIMI frame counts between patient groups with and without coronary slow flow phenomenon

\begin{tabular}{lccc}
\hline TIMI frame count & $\begin{array}{c}\text { CSF Group } \\
(\mathbf{n}=\mathbf{5 0})\end{array}$ & $\begin{array}{c}\text { Control Group } \\
(\mathbf{n}=\mathbf{5 0})\end{array}$ & P value \\
\hline LAD (CLAD) & $45.4 \pm 2.9$ & $20.3 \pm 1.8$ & $<0.001$ \\
\hline CX & $39.2 \pm 4.3$ & $18.8 \pm 1.3$ & $<0.001$ \\
\hline RCA & $41.1 \pm 3.7$ & $19.7 \pm 1.6$ & $<0.001$ \\
\hline
\end{tabular}

(LAD: Left anterior descending coronary artery, CSF: Coronary slow flow, CX:

Circumflex coronary artery, RCA: Right coronary artery)

Table 4. Comparison of aortic pressures and elastic properties between patient groups with and without coronary slow flow (CSF) phenomenon

\begin{tabular}{lccc}
\hline Aortic Parameters & $\begin{array}{c}\text { CSF } \\
(\mathbf{n}=50)\end{array}$ & $\begin{array}{c}\text { Controls } \\
(\mathbf{n}=50)\end{array}$ & $\begin{array}{c}\mathbf{P} \\
\text { Value }\end{array}$ \\
\hline Aortic systolic pressure, $(\mathrm{mmHg})$ & $118.8 \pm 12.6$ & $116.3 \pm 8.1$ & 0.236 \\
\hline Aortic diastolic pressure, $(\mathrm{mmHg})$ & $76.7 \pm 10.4$ & $75.2 \pm 7.5$ & 0.432 \\
\hline Aortic pulse pressure, $(\mathrm{mmHg})$ & $42.1 \pm 8.2$ & $41.2 \pm 7.1$ & 0.467 \\
\hline Aortic mean pressure, $(\mathrm{mmHg})$ & $90.7 \pm 10.6$ & $88.9 \pm 7.1$ & 0.317 \\
\hline Aortic fractional pulse pressure & $0.47 \pm 0.09$ & $0.46 \pm 0.09$ & 0.801 \\
\hline Aortic pulsatility index & $0.56 \pm 0.14$ & $0.55 \pm 0.13$ & 0.796 \\
\hline Aortic systolic diameter, $(\mathrm{mm})$ & $32.1 \pm 3.9$ & $31.9 \pm 3.9$ & 0.880 \\
\hline Aortic diastolic diameter, $(\mathrm{mm})$ & $28.9 \pm 4.5$ & $29.2 \pm 3.7$ & 0.701 \\
\hline Pulsatile aortic diameter change, $(\mathrm{mm})$ & $3.1 \pm 1.2$ & $2.7 \pm 0.9$ & 0.124 \\
\hline Aortic strain, $(\%)$ & $10.4 \pm 6.1$ & $9.6 \pm 3.7$ & 0.186 \\
\hline Aortic distensibility, $\left(10^{-6} \mathrm{~cm}^{2} \mathrm{dyn}^{-1}\right)$ & $8.1 \pm 4.5$ & $7.9 \pm 3.2$ & 0.838 \\
\hline Aortic stiffness index & $2.71 \pm 0.47$ & $2.82 \pm 0.34$ & 0.181 \\
\hline Aortic compliance, $\left(\mathrm{cm} / \mathrm{mmHg}^{2}\right.$ & $0.078 \pm 0.018$ & $0.071 \pm 0.023$ & 0.264 \\
\hline CSF: Coronary slow flow & \multicolumn{3}{l}{} \\
\hline
\end{tabular}

The hemodynamic parameters of aorta were compared between groups. Aortic pulse pressure ( $42.1 \pm 8.2$ vs. $41.2 \pm 7.1$ $\mathrm{mmHg} ; \mathrm{p}=0.467)$, aortic mean pressure $(90.7 \pm 10.6$ vs. $88.9 \pm 7.1 \mathrm{mmHg} ; \mathrm{p}=0.317)$, aortic fractional pulse pressure $(0.47 \pm 0.09$ vs. $0.46 \pm 0.09 ; \mathrm{p}=0.801)$, and aortic pulsatility index $(0.56 \pm 0.14$ vs. $0.55 \pm 0.13 ; \mathrm{p}=0.796)$ were similar between patients with CSF and controls (Table 4).

Elastic properties of ascending aorta were also compared between the groups. ASD $(32.1 \pm 3.9$ vs. $31.9 \pm 3.9 \mathrm{~mm}$; $\mathrm{p}=0.880), \operatorname{ADD}(28.9 \pm 4.5$ vs. $29.2 \pm 3.7 \mathrm{~mm} ; \mathrm{p}=0.701)$ and pulsatile aortic diameter change $(3.1 \pm 1.2$ vs. $2.7 \pm 0.9 \mathrm{~mm}$; $\mathrm{p}=0.124$ ) were similar between patients with CSF and controls. There was also no significant difference in terms of aortic strain (10.4 \pm 6.1 vs. $9.6 \pm 3.7 \%$; $=0.186$ ), aortic distensibility ( $8.1 \pm 4.5$ vs. $\left.7.9 \pm 3.210^{-6} \mathrm{~cm}^{2} \mathrm{dyn}^{-1} ; \mathrm{p}=0.838\right)$, aortic stiffness index ( $2.71 \pm 0.47$ vs. $2.82 \pm 0.34 ; \mathrm{p}=0.181)$, and aortic compliance $(0.078 \pm 0.018$ vs. $0.071 \pm 0.023 ; \mathrm{p}=0.264)$ between the groups (Table 4) (Figure 1).

\section{DISCUSSION}

In this study, we hypothesized that the mechanisms in the pathogenesis of CSF may also play role in the development of aortic atherosclerosis and increased aortic stiffness and aimed to evaluate the elastic properties of ascending aorta in patients with CSF. The results demonstrated that there was no significant difference in terms of hemodynamic parameters and elastic properties of ascending aorta between CSF patients and control group with a similar risk profile and demographic characteristics.

CSF is a well-known terminology by the interventional cardiologists in which opacification of major epicardial coronary arteries has been delayed at the distal segments without any atherosclerotic stenosis [19]. Besides its simple definition, the exact etiopathogenesis is unclear. However, various mechanisms have been suggested in the development of SCF including early atherosclerosis, inflammation, oxidative stress, impaired platelet function, coronary vasomotor dysfunction, and endothelial dysfunction [18-20].

The TFC technique has been successfully used for the assessment of coronary flow velocity using coronary angiograms. It is a simple, reproducible, objective, and quantitative index of coronary blood flow. In this method, the number of cineangiographic frames from initial contrast material opacification of the proximal portion of the coronary artery to opacification of the distal arterial landmarks with contrast material is counted ${ }^{17}$. TFC method has been widely used in the evaluation of CSF patients.

Aortic stiffness describes the elastic resistance that the aorta sets against its distension. In addition to being a blood carrying elastic artery, the aorta has important effects on left ventricular functions and coronary blood flow. The significance of the elastic features of vascular structures has been recognized in the development of atherosclerosis, and decreased elasticity is considered to be a factor causing atherosclerosis [21]. It was demonstrated that increased aortic stiffness and low aortic distensibility are indicators of impairment of the elastic structure of the aorta and associated with coronary artery disease [22]. 


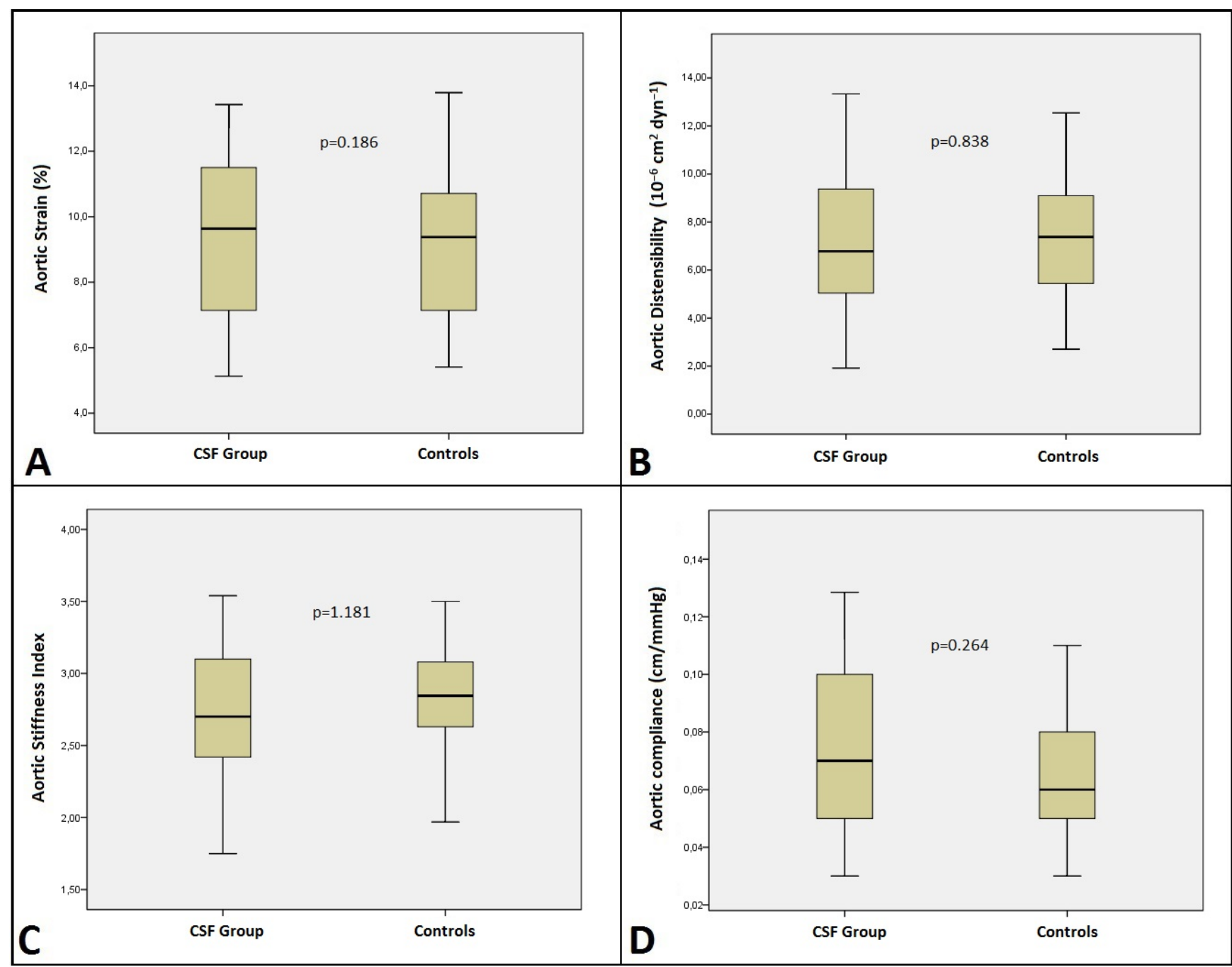

Figure 1. Comparison of box-plot graphs of aortic strain (A), aortic distensibility (B), aortic stiffness index (C) and aortic compliance (D) between patients with and without coronary slow flow (CSF)

A positive correlation has been reported between the amount of severity of atherosclerosis in the coronary bed and the aorta or its major branches [23]. Atherosclerotic changes in arterial wall include smooth muscle cell proliferation, deposition of lipid, and accumulation of collagen, elastin, and proteoglycans which have been known to structurally affect the elastic behavior of arterial walls [24].

Vascular elasticity is easily and accurately detectable with advanced technologies. Elastic features of the aorta can be measured with invasive methods, or noninvasively with echocardiography or specific devices measuring pulse wave velocity. Quantitative measurement of the elastic properties of the large arteries can be obtained by means of blood pressures and arterial diameters [25]. In our study, a noninvasive technique using blood pressures and aortic diameters measured by echocardiography was preferred.

In their study, Vizzardi et al found that aortic stiffness was significantly greater in patients with cardiac syndrome $\mathrm{X}$ than in normal subjects [26]. Cardiac syndrome $\mathrm{X}$ shares a similar pathophysiology with CSF phenomenon that includes the disorder of the microvasculature. This pathophysiological process of the disease may also affect the vasa vasorum because the blood flow of vasa vasorum is derived from the epicardial arteries. Therefore, we hypothesized that the mechanisms in the pathogenesis of CSF may also play role in the development of aortic atherosclerosis and increased aortic stiffness. So, we aimed to evaluate the elastic properties of ascending aorta in patients with CSF in this study. However, there was no significant difference in terms of hemodynamic parameters and elastic properties of ascending aorta between CSF patients and controls in our study. This may be caused by the limited number of patients in this study. A study involving more patients could have more significant results.

\section{CONCLUSION}

The results demonstrated that there was no significant difference in terms of hemodynamic parameters and elastic properties of ascending aorta between CSF patients and control group with a similar risk profile and demographic characteristics. Further large scale studies are required to determine the relationship between CSF and elastic properties of aorta. 
Declaration of interest: The authors report no conflicts of interest. Financial Disclosure: No financial support was received.

\section{REFERENCES}

1. Beltrame JF, Limaye SB, Horowitz JD. The coronary slow flowphenomenon - a new coronary microvascular disorder. Cardiology 2002;97:197-202.

2. Wang X, Nie S-P. The coronary slow flow phenomenon: characteristics, mechanisms and implications. Cardiovascular Diagnosis and Therapy 2011, 1(1):37-43.

3. Tatli E, Yildirim T, Aktoz M. Does coronary slow flow phenomenon lead to myocardial ischemia? Int J Cardiol. 2009;131(3): e101-e102.

4. Barutcu I, Sezgin AT, Sezgin N, et al. Increased high sensitive CRP level and its significance in pathogenesis of slow coronary flow. Angiology. 2007;58(4):401-407.

5. Enli Y, Turk M, Akbay R, et al. Oxidative stress parameters in patients with slow coronary flow. Adv Ther. 2008;25(1):37-44.

6. Mosseri M, Yarom R, Gotsman MS, Hasin Y. Histologic evidence for small-vessel coronary artery disease in patients with angina pectoris and patent large coronary arteries. Circulation. 1986; 74(5):964-972.

7. Gokce M, Kaplan S, Tekelioglu Y, Erdoğan T, Küçükosmanoğlu M. Platelet function disorder in patients with coronary slow flow. Clin Cardiol. 2005;28(3): 145-8.

8. Sezgin AT, Sigirci A, Barutcu I, et al. Vascular endothelial function in patients with slow coronary flow. Coron Artery Dis. 2003; 14(2):155-161.

9. Chon JN. Arterial compliance to stratify cardiovascular risk: more precision in therapeutic decision making. Am J Hypertens. 2001;14:258S-63S.

10. Boutouyrie P, Tropeano AI, Asmar R, et al. Aortic stiffness is an independent predictor of primary coronary events in hypertensive patients: a longitudinal study. Hypertension 2002; 39: 10-15.

11. Stefanidis C, Stratos C, Boudoulas H, Vlachopoulos C, Kallikazaros I, Toutouzas P. Distensibility of the ascending aorta in coronary artery disease and changes after nifedipine administration. Chest 1994;105:101723.

12. Epstein SE, Cannon III RO. Site of increased resistance to coronary flow in patients with angina pectoris and normal epicardial coronary arteries. J Am Coll Cardiol. 1986;88:459-61.

13. Stefanadis C, Stratos C, Boudoulas H, Kourouklis C, Toutouzas P. Distensibility of the ascending aorta: comparison of invasive and non-invasive techniques in healthy men and in men with coronary artery disease. Eur Heart J. 1990;11:990-996.
14. O'Rourke MF, Staessen JA, Vlachopoulos C, Duprez D, Plante GE. Clinical applications of arterial stiffness; definitions and reference values. Am J Hypertens. 2002;15:426-444.

15. Vitarelli A, Giordano M, Germanò G, et al. Assessment of ascending aorta wall stiffness in hypertensive patients by tissue Doppler imaging and strain Doppler echocardiography. Heart. 2010;96:1469-1474.

16. Friedewald WT, Levy RI, Fredrickson DS. Estimation of the concentration of low-density lipoprotein cholesterol in plasma, without use of the preparative ultracentrifuge. Clin Chem. 1972;18(6):499-502.

17. Gibson CM, Cannon CP, Daley WL, et al. TIMI frame count: a quantitative method of assessing coronary artery flow. Circulation 1996;93:879-88.

18. Pekdemir H, Cin VG, Cicek D, et al. Slow coronary flow may be a sign of diffuse atherosclerosis. Contribution of FFR and IVUS. Acta Cardiol 2004; 59(2):127-133.

19. Tambe AA, Demany MA, Zimmerman HA, Mascarenhas E. Angina pectories and slow flow velocity of dye in coronary arteries-A new angiografic finding. Am Heart J. 1972; 84: 66-71.

20. Nissen SE, Gurley JC, Grines CL, et al. Intravascular ultrasound assessment of lumen size and wall morphology in normal subjects and patients with coronary artery disease. Circulation 1991; 84(3):108799.

21. Levent E, Ozyurek AR, Ulger Z. Evaluation of aortic stiffness in tobacco-smoking adolescents. J Adolesc Health. 2004;34:339-343.

22. Pitsavos C, Toutouzas K, Dernellis J, et al. Aortic stiffness in young patients with heterozygous familial hypercholesterolemia. Am Heart J 1998;135:604-648.

23. Isii T, Newman WP III, Guzman MA, Hosoda Y, Strong JP. Coronary and aortic atherosclerosis in young men from Tokyo and New Orleans. Lab Invest. 1986;54:561565.

24. Hirai T, Sasayama S, Kawasaki T, Yagi S. Stiffness of systemic arteries in patients with myocardial infarction. A noninvasive method to predict severity of coronary atherosclerosis. Circulation 1989 Jul;80(1):78-86.

25. Kawasaki T, Sasayama S, Yagi S, Asakawa T, Hirai T. Noninvasive assessment of the age related changes in stiffness of major branches of the human arteries. Cardiovasc Res. 1987;21:678-687.

26. Vizzardi E, Trichaki E, Bonadei I, et al. Elastic aortic properties in patients with $\mathrm{X}$ syndrome. Heart Lung Circ. 2014 Feb;23(2):114-8. 